\title{
$\beta$ Cephei Pulsation Anomalies: Potential New Windows into the Instabilities and Evolution of Early B Stars
}

\author{
B.A. Goldberg ${ }^{1}$, R.S. Polidan ${ }^{2}$, R.A. Crowe ${ }^{3}$, G.C.L. Aikman ${ }^{4}$, \\ R.J. Bambery ${ }^{1}$, J.T. Gathright ${ }^{3}$, G.J. Odgers ${ }^{4}$ \\ ${ }^{1}$ Jet Propulsion Laboratory/Caltech, Pasadena, CA, U.S.A., ${ }^{2}$ NASA Goddard Spaceflight \\ Center, Greenbelt, MD, U.S.A., ${ }^{3}$ University of Hawaii at Hilo, HI, U.S.A., ${ }^{4}$ Dominion \\ Astrophysical Observatory, Victoria, BC, Canada
}

\begin{abstract}
We have obtained Voyager Ultraviolet Spectrometer (UVS) measurements of wellknown $\beta$ Cephei stars, which now total more than 1500 hours $(>300$ pulsation cycles!) and which constitute the most comprehensive coherent data set that can address fundamental pulsation properties of a significant cross-section of the group. The extended measurement sequences for individual stars, which cover many successive pulsation cycles at wavelengths where pulsation amplitudes reach a maximum, can provide more comprehensive tests of pulsation stability than any ground-based data. During 1990-91, we acquired more than 100 hours of ground-based high-resolution spectroscopic observations and UBV photometric observations, simultaneous and near-simultaneous with the UVS data set. Analysis has been initiated at NASA's Goddard Spaceflight Center (NASA/GSFC), the University of Hawaii at Hilo (UH Hilo), and the Dominion Astrophysical Observatory (DAO).
\end{abstract}

\section{Description of Voyager UVS and Ground-Based Data}

The two Voyager spacecraft have objective grating spectrometers with wavelength coverage of 500-1700 $\AA$. Spectral resolutions of approximately $18 \AA$ for point sources and $30 \AA$ for diffuse sources are achieved. Instrumental sensitivity is optimized for the $800-1200 \AA$ region. Typical limiting fluxes at $1050 \AA$ in the far-UV are $1.0 \times 10^{-12} \mathrm{ergs} \mathrm{cm}^{-2} \mathrm{sec}^{-1} \AA^{-1}$ for Voyager 1 (5.0 $\times 10^{-13}$ for Voyager 2). In-flight performance of the UV spectrometers has been reviewed by Broadfoot et al. (1981, Journal of Geophysical Research, 86, p. 8259). During 1990-91, we obtained UVS data on the stars BW Vulpeculae, $\beta$ Cephei, $\nu$ Eridani, $\delta$ Ceti and 12 Lacertae.

Measurements at the DAO were made using CCD sensors on both the Cassegrain spectrograph of the 1.8-meter telescope and the coudé spectrograph of the 1.2-meter telescope. Spectral resolutions were approximately $0.3 \AA$ and $0.1 \AA$ respectively; time resolution was typically in the range of $2-4$ minutes with a $\mathrm{S} / \mathrm{N}$ exceeding 50:1. The lines of Si III, He I, $\mathrm{Mg}$ II, O II and C III in the wavelength range 4450-4600 $\AA$ were among those observed. For observations with the UH 2.2-meter telescope on Mauna Kea, a CCD sensor was used on the coudé spectrograph. The spectral resolution was $0.2 \AA$ and the time resolution was 10 15 minutes with a $\mathrm{S} / \mathrm{N}$ in the range $100-150$. In addition, simultaneous UBV photometric 
observations were obtained on the UH 0.6-meter "Air Force" telescope during May 1991. The ground-based program has been an outstanding success, having produced almost 100 hours of simultaneous data on the stars BW Vul, $\beta$ Cep, $\nu$ Eri, $\delta$ Cet and 12 Lac.

\section{Scientific Objectives and Analysis}

The scientific objectives which can be addressed directly with the available data are as follows: (1) determine the origin and significance of an "instability anomaly" present in the far-UV light curves of $\beta$ Cep and $\nu$ Eri; (2) determine cycle-to-cycle pulsational stability from Voyager UVS data; (3) determine the role of atmospheric shock waves; investigate the relationship of shock waves to proposed pulsation mechanisms and to the range in behaviour within the group; (4) determine whether there exist unique relationships between pulsation amplitude and line profile variations, light and velocity curve shapes, etc.; (5) institute an analysis to discriminate radial from non-radial pulsation modes using temperatures derived from the Voyager UVS data; (6) continue the analysis of the long-term (decades) pulsational stability of stars such as BW Vul and assess the evolutionary significance. The more general program goals are as follows: (a) further define the evolutionary status of $\beta$ Cephei stars; (b) explain the wide range in pulsation amplitude within the $\beta$ Cephei group; (c) identify, if possible, the pulsation mechanism; (d) determine the significance of $\beta$ Cephei instability within the context of the variability of early B stars.

Data analysis is currently in progress at NASA/GSFC, UH Hilo, and DAO. Examples of Voyager UVS light curves are given below. Observations have been folded into one cycle. The "instability anomaly", shown for $\beta$ Cep in Fig. 2, is manifested in the form of unexplained fluctuations in intensity near the phase of maximum UV flux. In Fig. 1, UVS data for $\beta$ Cep obtained during May 1991 show that the fluctuations near maximum are still present a decade later. Moreover, the magnitude of the fluctuations during 1991 appears to be comparable to that found in the 1981 data.
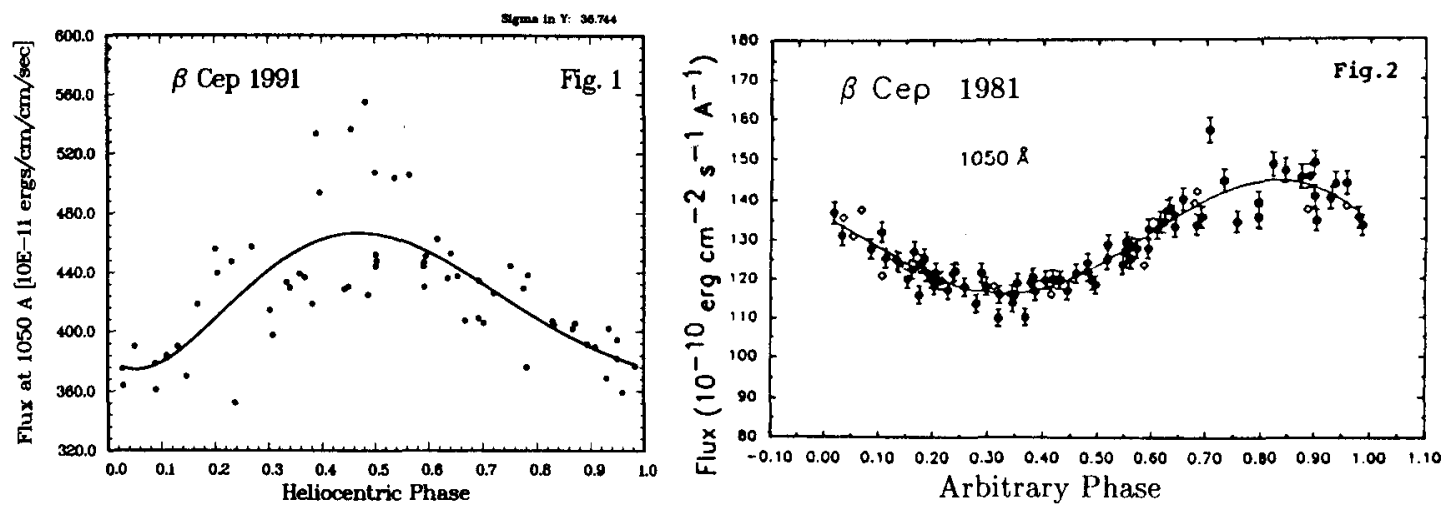

Fig. 1: Voyager UVS observations of $\beta$ Cep $(P=4 \mathrm{~h} 34 \mathrm{~m})$ taken over a 5-day observing period in 1991. Fig. 2: Voyager UVS observations of $\beta$ Cep taken over a 26-hour observing period in 1981 (Rautenkrantz and Polidan, in prep.); note the "instability anomaly" near maximum light. 